\title{
Interaction of magnetic field systems leading to an X1.7 flare due to large-scale flux tube emergence
}

\author{
H. Li ${ }^{1}$, B. Schmieder ${ }^{2}$, M. T. Song ${ }^{1}$, and V. Bommier ${ }^{3}$ \\ 1 Purple Mountain Observatory, Chinese Academy of Sciences, Nanjing 210008, PR China \\ e-mail: lihui@mail.pmo.ac.cn \\ 2 LESIA, Observatoire de Paris, Section de Meudon, 92195, Meudon Principal Cedex, France \\ 3 LERMA, Observatoire de Paris, Section de Meudon, 92195, Meudon Principal Cedex, France
}

Received 18 March 2007 / Accepted 3 September 2007

\begin{abstract}
Aims. The aim of this paper is to understand the magnetic configuration and evolution of an active region, which permitted an X1.7 flare to be observed during the decaying phase of a long-duration X1.5 flare on 2005 September 13.

Methods. We performed a multi-wavelength analysis using data from space-borne (Solar and Heliospheric Observatory (SOHO), Transition Region and Coronal Explorer (TRACE), Reuven Ramaty High-Energy Solar Spectroscopic Imager (RHESSI), GOES) and ground-based (the French-Italian THEMIS telescope and the Huairou Video Vector Magnetograph (HVVM)) instruments. We coaligned all the data in order to study the origin of the flare by comparing the observed magnetic field structures with the emissions detected by different instruments.

Results. Reconstructed RHESSI images show three hard X-ray (HXR) sources. In TRACE $195 \AA$ Amages, two loops are seen: a short bright loop and a longer one. Five ribbons are identified in $\mathrm{H} \alpha$ images, with two of them remnant ribbons of the previous flare. We propose the following scenario to explain the X1.7 flare. A reconnection occurs between the short loop system and the longer loops (TRACE $195 \AA$ ). Two X-ray sources could be the footpoints of the short loop, while the third one between the two others is the site of the reconnection. The $\mathrm{H} \alpha$ ribbons are the footprints in the chromosphere of the reconnected loops. During the reconnection, the released energy is principally nonthermal according to the RHESSI energy spectrum analysis (two orders of magnitude higher than the maximum thermal energy). The proposed scenario is confirmed by a nonlinear force-free field (NLFFF) extrapolation, which shows the presence of short sheared magnetic field lines before the eruption and less sheared ones after the reconnection, and the connectivity of the field lines involved in the flaring activity is modified after the reconnection process. The evolution of the photospheric magnetic field over a few days shows the continuous emergence of a large-scale magnetic flux tube, the tongue-shape of the two main polarities of the active region being the signature of such an emergence. After the previous X1.5 flare, the emergence of the tube continues and favors new magnetic energy storage and the onset of the X1.7 flare.
\end{abstract}

Key words. sun: flares - sun: X-rays, gamma rays - sun: magnetic fields

\section{Introduction}

The source of energy released in solar flares is generally believed to be the free energy stored in nonpotential magnetic structures. The energy can suddenly be released through magnetic reconnection leading to flares. The conditions favoring the production of big solar flares include (i) complex magnetic configuration of the active region, such as the so-called $\delta$ configuration in which two umbrae of opposite polarity share a common penumbra, as reviewed by Schmieder \& van Driel-Gesztelyi (2005); (ii) a high level of shear, twist, or emerging flux; and (iii) magnetic field strength in the corona that influences the strength of the X-ray flare. Emergence of a magnetic field can occur on a large scale (shear, e.g., Hagyard et al. 1984; Li et al. 2000a) and small scale (emergence of a small bipole, e.g., Berlicki et al. 2004). Various methods have been developed to predict solar flares including statistics or systematic detection of parameter changes such as shear, tilt, strength of the magnetic field (e.g., Li et al. 2000a,b; Falconer 2001; Leka \& Barnes $2003 a, b)$. Indirect methods based on determing of the magnetic configuration of an active region have been under development for more than 40 years (e.g., Schmidt 1964; Semel \& Rayrole 1968; Chiu \& Hilton 1977; Alissandrakis 1981; Sakurai 1982;
Démoulin et al. 1997; Song et al. 2006). If any current in a region is confined to heights at or below the photosphere, the field above is a current-free field (CFF); if not, a force-free field configuration $(\boldsymbol{J} \times \boldsymbol{B}=0, \boldsymbol{\nabla} \times \boldsymbol{B}=\alpha \boldsymbol{B})$ is commonly assumed because of the low plasma $\beta$ in the solar atmosphere, corresponding to $\boldsymbol{J}$ parallel to $\boldsymbol{B}$ (Metcalf et al. 1995). If $\alpha$ is assumed to be constant in all the active region, we get a linear force-free field (LFFF) configuration. So far, extrapolations using CFF and LFFF configurations are commonly used because they are easy to compute with respect to nonlinear force-free field (NLFFF) extrapolations. LFFF extrapolations only need the longitudinal photospheric field, while NLFFF need the magnetic field vector at each point in the photosphere for the boundary conditions. An NLFFF can relax to an LFFF with the same magnetic helicity (Berger 1984; Taylor 1986; Parker 1989; Aly 1992; Nandy et al. 2003) during an eruptive MHD process. For a complicate flareproductive $\delta$ region, NLFFF configuration may nevertheless be a more reasonable, reliable, and promising approximation compared to CFF and LFFF configurations (Song et al. 2006, 2007; Régnier \& Priest 2007). It is observed that the magnetic field is nearly force-free in the corona and far from force-free in the lower photosphere (below about $400 \mathrm{~km}$ ) (Metcalf et al. 1995; Moon et al. 2002). 
Many multi-wavelength studies have been performed to understand where the site of the eruption is and the reason for its onset using these different methods (e.g., Aulanier et al. 2000; Galsgaard et al. 2000; Fletcher et al. 2001a,b; Titov et al. 2003; Parnell \& Galsgaard 2004; Aulanier et al. 2005; Galsgaard \& Parnell 2005; Aulanier et al. 2006; Régnier \& Canfield 2006). The diversity of the answers actually shows that this question is still open. Theoretical and numerical simulations are recently another approach to understand the mechanism of flare onsets. Boundary conditions are needed for this approach, and only observations can give such constraints on the theoretical models. For example, numerical simulations have shown that interactions of the new emerging flux with pre-existing corona magnetic fields lead to the onset of coronal mass ejections (CMEs), solar flares, and coronal X-ray jets (e.g., Chen \& Shibata 2000; Yokoyama \& Shibata 2001; Miyagoshi \& Yokoyama 2004).

Accumulation of observations and detailed study of an individual event is still necessary for understanding the importance of an emerging flux, on both small and large scales, in triggering solar eruptions and incorporating numerical simulations.

The released energy in a flare is converted into thermal and kinetic energy in the corona, leading to particle acceleration, direct heating of flare plasma, and plasma motions. The so-called thermal and nonthermal models deal with two extreme cases of flare models. Thermal models assume that most of the released energy goes into the impulsive heating of the plasma near the flare site (Datlowe \& Lin 1973; Brown et al. 1979; MacKinnon $\&$ Brown 1984). Conduction fronts are formed in the loops and move at a fast speed (typically $100-1000 \mathrm{~km} \mathrm{~s}^{-1}$ ) towards the chromosphere in a few seconds $(10 \mathrm{~s})$. In contrast, the nonthermal thick-target models assume that most of the released energy goes into the acceleration of particles (Brown 1971, 1972; Brown \& MacKinnon 1985). The thick-target model is more reasonable when the energy release site is in the low solar atmosphere where the plasma density is high. Models consisting of thermal component, nonthermal component, or both have been used to study the energy contents in solar flares (e.g., McDonald et al. 1999; Berlicki et al. 2004; Li et al. 2005). The thermal component represents the flare energy contained in the thermal plasma, while the nonthermal one can give us some suggestions about the importance of nonthermal particles in the overall energy budget of solar flares. The thermal and nonthermal energies in solar flares obtained so far change from case to case (McDonald et al. 1999; Berlicki et al. 2004; Li et al. 2005; Li $\&$ Li 2007). Therefore, case study is still important and necessary for understanding energy content in solar flares.

In this paper, we study the X1.7 flare of 2005 September 13 at 23:22 UT that took place in active region NOAA 10808 and its associated coronal mass ejection (CME) based on the collected data from multi-instrument observations. The Reuven Ramaty High Energy Solar Spectroscopic Imager (RHESSI, Lin et al. 2002) observations allowed us to reconstruct the energy spectrum in the range of 3-200 keV for this flare, which can be reproduced by calculations based on theoretical models. We have investigated the magnetic configuration responsible for this flare and the thermal and nonthermal energy content in the flare. We introduce our observations and data in Sect. 2 and describe the magnetic configuration of the active region in Sect. 3. We present our interpretation of the flare observations in Sect. 4 and the computation of the magnetic field lines over the active region using an NLFFF approach in Sect. 5. We finally give a possible scenario to explain the onset of this flare based on the observations and the extrapolations (Sect. 6).

\section{Instruments and observations}

The X1.7 flare took place in the flare-productive active region NOAA 10808 when it was close to the solar disc center. This active region has already been studied by different groups, but they concentrated mainly on the eruption of a large filament located on the periphery of the active region (Wang et al. 2007; Nagashima et al. 2007)

The X1.7 flare was observed during a multi-wavelength observation campaign (JOP 178) by space-borne and groundbased instruments, including GOES, RHESSI, the Solar and Heliospheric Observatory (SOHO) (Domingo et al. 1995), the Transition Region and Coronal Explorer (TRACE) (Handy et al. 1999), THEMIS on the Canary Islands in the multi-line spectroscopy mode (MTR, Rayrole \& Mein 1993), and the Huairou Video Vector Magnetograph (HVVM) mounted on the Solar Magnetic Field Telescope (SMFT) at Huairou Solar Observing Station (HSOS) (Ai \& Hu 1986), which is a part of the MultiChannel Solar Telescope (Deng et al. 1997).

The Solar Geophysical Data (SGD) register indicates that the X-ray flare (X1.7) started at 23:15 UT, peaked at 23:22 UT, and ended at 23:30 UT (Fig. 1, left panel). The SGD has no registration about the $\mathrm{H} \alpha$ flare. An X-ray flare occurred before this flare with two peaks at 19:27 UT and at 20:04 UT. It is a longduration event and its X-ray emission lasted rather a long time so that the X1.7 flare occurred in the decreasing phase of its $\mathrm{X}$-ray emission. Therefore, they actually formed a triple X-ray event. The EUV data of this flare in $195 \AA$ were from the observations of TRACE. The full-disk intensity images and magnetic field data, which are used to coalign the ground-based and spaceborne observations, were obtained with the Michelson Doppler Imager (MDI) (Scherrer et al. 1995) onboard SOHO. The H $\alpha$ filtergrams were obtained at the line-center with the $\mathrm{H} \alpha$ telescope at HSOS. Hard X-ray (HXR) data used in this paper were from RHESSI.

The RHESSI observation covers the whole flaring process. We retrieved RHESSI light curves in five energy channels, i.e., 6-12 keV, $12-25 \mathrm{keV}, 25-50 \mathrm{keV}, 50-100 \mathrm{keV}$, and 100-300 keV. The retrieved light curves are shown in Fig. 1 (right panel). We reconstructed RHESSI images in the five energy channels above from collimators and detectors $3 \mathrm{~F}-6 \mathrm{~F}$ and $8 \mathrm{~F}$ using the CLEAN image algorithm with a time interval of $20 \mathrm{~s}$. We also retrieved an HXR energy spectrum from RHESSI observation to study the energy content in the flare. More details about this and the energy spectrum analysis will be given in Sect. 4.3.

This active region was observed by THEMIS in MTR mode in the period of 14:25-15:25 UT on September 13 in the Fe I $6301.5 \AA$ and $6302.5 \AA$ doublet. THEMIS MTR observing mode allows polarimetric observations of the Sun in multiple spectral regions simultaneously. A detailed description of THEMIS/MTR instrumentation can be found in López Ariste et al. (2000, 2006). During the observation, the slit was $0.5^{\prime \prime}$ wide and oriented along the local solar meridian with the raster movement parallel to the equator. The pixel size along the slit is $0.4^{\prime \prime}$. For each raster position, 6 acquisitions were taken with changing polarimeter configurations to complete a modulation cycle, which allows recovery of the Stokes parameters. After correcting the dark-current and flat-field, the raw data were combined to extract the Stokes parameters by applying the beam exchange method. And then the UNNOFIT inversion code (Landolfi et al. 1984) improved by introducing a magnetic filling-factor parameter (Bommier et al. 2007) was used to derive the magnetic field from the observed Stokes 

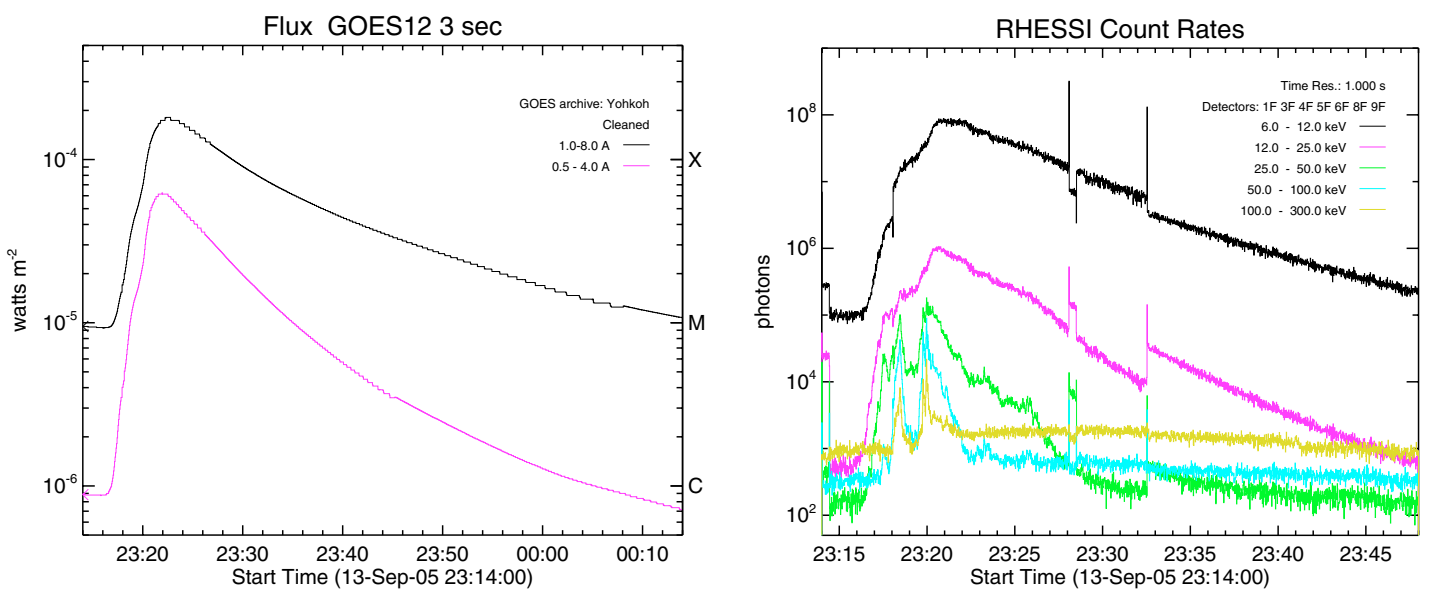

Fig. 1. Left: GOES X-ray flux in $0.5-4 \AA$ and $1-8 \AA$. Right: RHESSI light curves in the energy range of 6-12 keV, 12-25 keV, 25-50 keV, $50-100 \mathrm{keV}$, and $100-300 \mathrm{keV}$ retrieved from the RHESSI data of all the nine front detectors except $2 \mathrm{~F}$ and $7 \mathrm{~F}$.

profiles. The magnetic and non-magnetic theoretical atmospheres, mixed in the proportion given by the filling factor, were derived from the same set of parameters, except for the presence (or absence) of a magnetic field. Readers are referred to Bommier et al. (2007) for details about the inversion code.

The HVVM was established in the 1980s (Ai \& Hu 1986), has been updated in recent years, and now has a field-of-view (FOV) of $225^{\prime \prime} \times 170^{\prime \prime}$ with a pixel resolution of $0.35^{\prime \prime}$. The pixel resolution can be changed to another required value by binning the data in the calibration process. The vector magnetic fields are derived from the measurements of the four Stokes parameters $I, Q, U$, and $V$. The transverse fields (parameters $Q$ and $U$ ) were observed at the Fe I 5324.19 $\AA$ line center, and the lineof-sight (LOS) component (parameter $V$ ) at $0.075 \AA$ from the Fe I $5324.19 \AA$ line center. The calibration of the Huairou Vector Magnetograph has been discussed in Ai et al. (1982), Wang et al. (1996), and $\mathrm{Li}$ (2002). The noise level of the original magnetograms is usually about $10 \mathrm{G}$ for the LOS component and about $100 \mathrm{G}$ for the transverse one ( $\mathrm{Li} 2002)$.

We use the algorithm of Wang et al. (2001) to remove the $180^{\circ}$ ambiguity of the transverse field obtained with THEMIS/MTR and HVVM. This algorithm compares the observed field to an LFFF computed using a Fourier transform method with the observed line-of-sight field as the boundary condition.

\section{Magnetic configuration}

\subsection{Magnetic feature of the active region}

The X1.7 flare was located around S10E03 in active region NOAA 10808. Figure 2 shows the vector magnetograms of the active region obtained with HSOS/HVVM (Frame (a)) and THEMIS with MTR mode in the Fe I 6301.5 $\AA$ and $6302.5 \AA$ doublet on September 13 in the period of 14:25-15:25 UT (Frame (b)). To show the strong shear near the magnetic polarity inversion line (PIL) more clearly in Fig. 2b, we only present a part of the full THEMIS/MTR field map, which covers the $\delta$ spot and its nearby region. As shown in Fig. 2a, this active region consists of a main $\delta$ spot, which is surrounded by several groups of satellite sunspots. Two sunspot groups of positive polarity are located to the east of the $\delta$ spot, and one group of negative polarity to the west. A magnetic PIL passes through the middle of the $\delta$ spot, around which large magnetic shear is present (Fig. 2b). THEMIS/MTR polarimetric observation has higher sensitivity and accuracy than the HSOS/HVVM and shows the strong shear more clearly around the magnetic PIL (Fig. 2b). It is expected that current exists in the upper left between the spot shear (when the vectors are parallel to the $\mathrm{X}$-axis) and current sheet around the PIL (when the vectors have complete different angle on the two sides).

Figure 2c presents the LOS magnetic field with the main inversion line observed with HVVM, and Fig. 2d the difference of the magnetic vector orientations before and after the X1.7 flare. However, these vectors are difficult to compare directly with THEMIS vectors, which were registered more than eight hours before. During the eight hours, several X-class flares occurred. In Fig. $2 d$ the direction of the arrows indicates the azimuth angle and their length indicates the magnitude of the transverse field. This figure clearly presents the flare-related changes of the transverse field in both magnitude and direction in the flaring region close to the magnetic PIL passing through the $\delta$ spot (see the ellipse region in Fig. 2d), indicating the possible changes of magnetic connectivity.

\subsection{Evolution of the magnetic field}

Figure 3 summarizes the evolution of the LOS magnetic field extracted from the MDI observations from 2005 September 9 to 14 . The main positive and negative polarities underwent a fast counterclockwise rotation. The line connecting the centers of the two main magnetic polarities was rotated counterclockwise by $87^{\circ}$ from $22: 24$ UT of 11 September (Fig. 3c) to 14:27 UT of 2005 September 14 (Fig. 3f), namely, the average rotating angular speed is $1.36^{\circ}$ per hour. The positive/negative polarity is elongated towards the west/east with "tongues". This pattern is a characteristic feature of a long-term twisted emerging flux as described by López Fuentes et al. (2000) (see their Fig. 5). The series of magnetograms shows the classical appearance of a bipole followed by the separation of the two opposite magnetic polarities as observed for emergence of an untwisted $\Omega$ loop. However, when the flux tube is twisted, an asymmetry appears in the magnetogram because of the contribution of the azimuthal component to the observed vertical component of the field. This azimuthal component produces two elongated polarities (tongues), which extend between the main ones. The strength of these tongues is directly proportional to the magnitude of the twist, and their position depends on the sign (positive twist in our case). The tongues are only present when the apex 

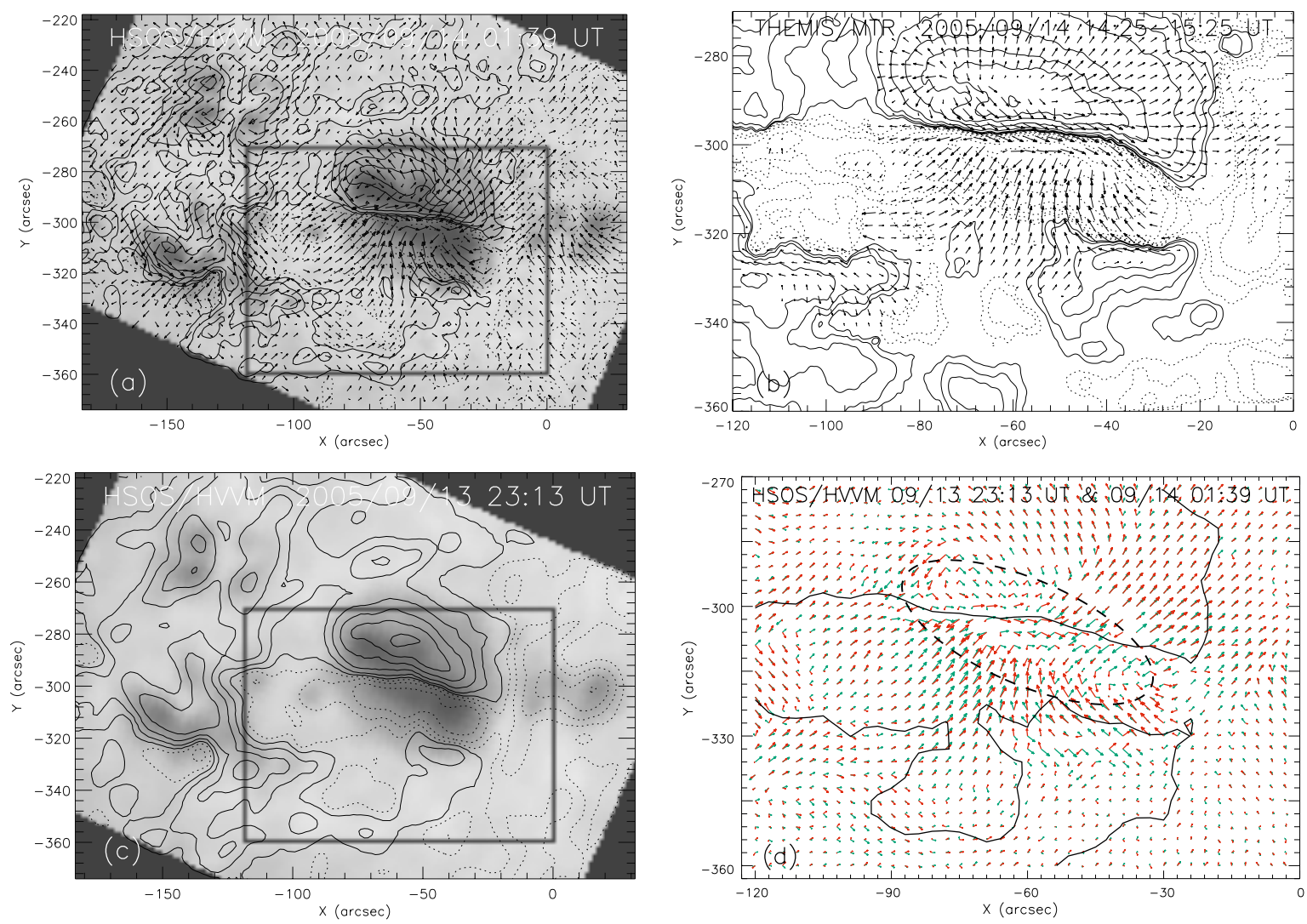

Fig. 2. Vector magnetograms of active region NOAA 10808 obtained a) with HVVM at 01:39 UT of September 14, and b) with THEMIS in the MTR observing mode in the Fe I 6301.5 $\AA$ and 6302.5 $\AA$ line on September 13 in the period of 14:25-15:25 UT. c) LOS magnetogram obtained with HVVM at 23:13 UT on September 13. d) Comparison of the transverse fields obtained with HVVM at 23:13 UT on September 13 (green arrows) and at 01:39 UT on September 14 (red arrows). The length of the arrow is proportional to the magnitude of the transverse fields. The solid lines indicate the magnetic neutral lines, and the dashed ellipse encompassing the region where the transverse field underwent significant changes. The background images in Frames a) and c) are the corresponding photospheric images. The rectangular boxes in Frames a) and c) have the same size and represent the FOV of THEMIS shown in Frame b) and that shown in Frame d) for the transverse field comparison. The contour levels of the magnetic field are $\pm 50,200,500,1000,1600,2200,2800 \mathrm{G}$. North is at the top and east to the left. Solid/dotted contours indicate a positive/negative magnetic field. We will keep this convention hereafter for all images in the paper.

of the flux tube is crossing the photosphere during the flux emergence. This implies the presence of a sigmoid as observed by Nagashima et al. (2007).

\section{Flare and CME}

\subsection{The $H \alpha$ and EUV flare}

We present five $\mathrm{H} \alpha$ filter images from HSOS taken at its line center in Fig. 4 and mark the $\mathrm{H} \alpha$ bright patches with B1 through B5. Superposed as contours in Fig. 4a are the TRACE $195 \AA$ image at 23:22:09 UT, and that in Fig. 4b are the RHESSI image at 23:18:20 UT in 50-100 $\mathrm{keV}$ and $\mathrm{H} \alpha$ image at 23:18:08 UT. We notice from the figure that the $\mathrm{H} \alpha$ flare started around 23:18 UT from B1 and B2 (Fig. 4a), which first appeared as two weak brightenings on the two sides of the magnetic PIL (Fig. 3e). Both B3 and B4 are remnant bright patches of the previous X1.5 flare. The area and brightness of B1 and B2 increased rapidly, and due to their expansion, they merged so that we can no longer distinguish them (Figs. $4 \mathrm{~b}-\mathrm{d}$ ). The mixed bright patch joined the remnant brightening B3 after 23:28 UT. After its appearance around 23:20 UT (Fig. 4b), B5 expanded quickly and finally joined B4. Superposed on Fig. $4 \mathrm{c}$ as contours is the LOS magnetic field from MDI at 22:23 UT of September 13, which allowed us to see the locations of $\mathrm{H} \alpha$ kernels with respect to the magnetic PIL.

Four TRACE $195 \AA$ images are shown in Fig. 5. These images show an elongated and tiny strong emission area and a weaker emission area (marked with "WB" in Fig. 5d) to the west of the strong brightening. The strong emission looks like a lowlying compact flare loop, which strides over the magnetic PIL and roots in opposite magnetic polarities, and the weak emission well enhanced around 23:37 UT could be a part of a longer loop cut because of the limited field-of-view. The TRACE $195 \AA$ A tiny loop connects $\mathrm{H} \alpha$ ribbons B1 and B2 (Fig. 4a).

We also superpose the TRACE $1600 \AA$ image of the previous X1.5 flare at 20:10 UT on its TRACE $195 \AA$ at 23:10 UT in Fig. 6 (thin contours). The figure tells us that the X1.5 flare has several ribbons in $1600 \AA$, which are located far away from each other. That means this flare involved large-scale magnetic topology. Alignment of these flare ribbons with magnetograms indicates that this X1.5 flare was a quadrupolar flare with far away ribbons. The remnant brightenings of the flare in $\mathrm{H} \alpha$ shown in Fig. 4 provide some circumstantial evidence for this point. Large systems of post-flare loops of the previous X1.5 flare are still visible during the $\mathrm{X} 1.7$ flare with no visible interaction with the newly bright loops (Fig. 6). Detailed study of the previous flare is beyond the scope of this paper. 

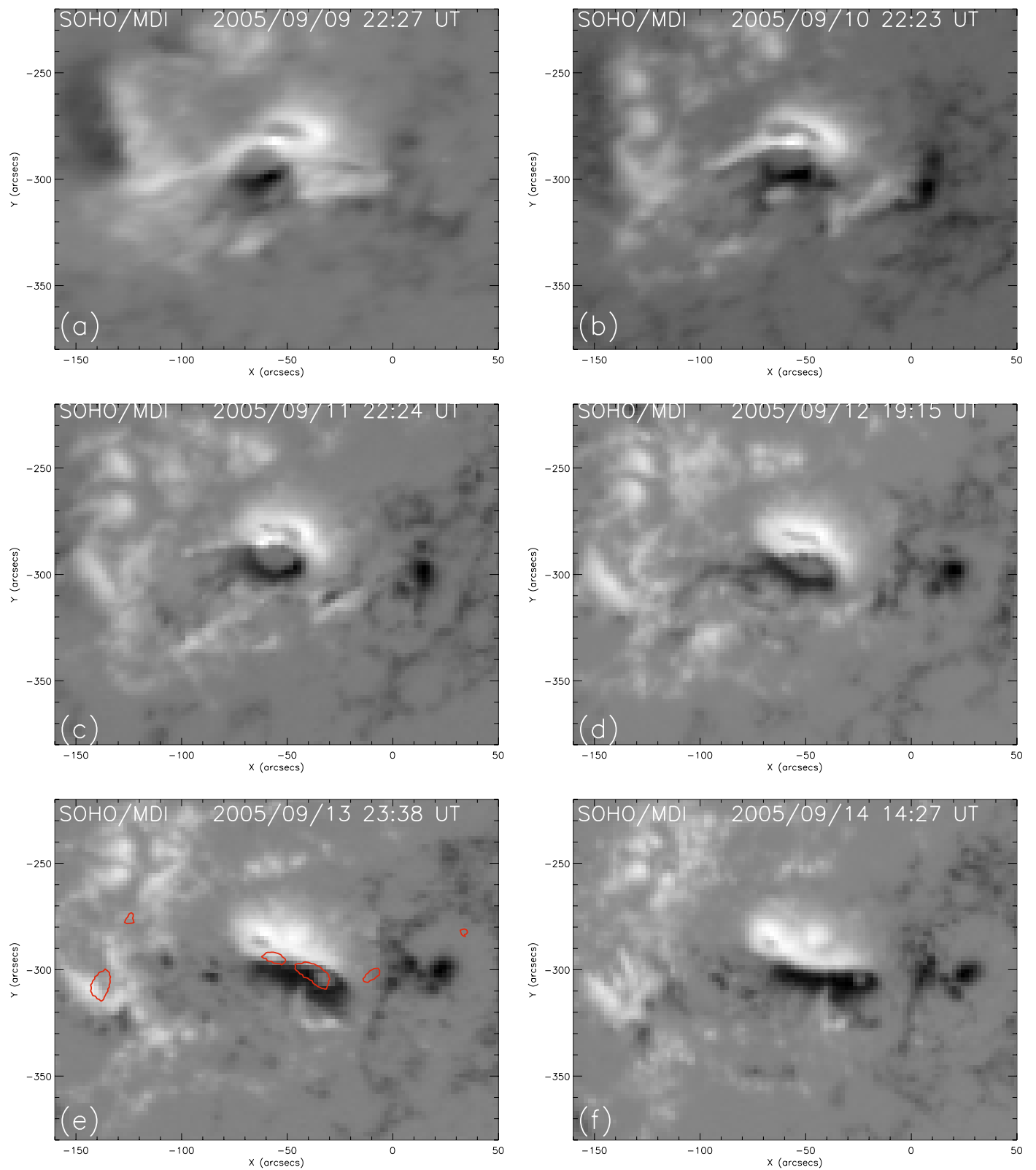

Fig. 3. Selected MDI LOS magnetograms for the active region from September 9 to 14. Observation times are shown in each image. White stands for positive polarity and black for negative one. Superposed as contours in Frame e) is the H $\alpha$ image at 23:20:06 UT from HSOS. Note that the images are corrected for the solar differential rotation to 22:00 UT of September 13.

\subsection{The HXR flare}

The RHESSI light curves in Fig. 1 (right panel) show three main peaks in all energy channels, which are most evident in the $25-50 \mathrm{keV}$ and $50-100 \mathrm{keV}$ energy channels with a time separation about $50 \mathrm{~s}$ and $80 \mathrm{~s}$. This could be due to magnetic reconnections occurring intermittently during the flaring process, which result in multiple energy release events. We will call the three peaks P1, P2, and P3 hereafter, which are in our defined time bins (see description below) of 23:17:20-23:17:40 UT, 23:18:20-23:18:40 UT, and 23:19:40-23:20:00 UT, respectively.

We reconstructed RHESSI HXR images using the CLEAN algorithm in five energy channels (Sect. 2). A selection of retrieved RHESSI images at different times and energy channels are presented in Fig. 7. RHESSI observed X-ray emission of this flare first at 23:16:20-23:16:40 UT in the $12-25 \mathrm{keV}$ energy channel, while the $6-12 \mathrm{keV}$ energy channel also shows weak emission. HXR emission only below $50 \mathrm{keV}$ was detected before 23:18:00 UT (Fig. 7, rows 1 and 2) and it appeared as a loop-top compact source (Fig. 7).

HXR emission in energy up to $200 \mathrm{keV}$ was detected during the periods from 23:18:00 UT to 23:18:40 UT and from 23:19:40 UT to 23:20:20 UT. RHESSI images at 23:18:20-23:18:40 UT in the $25-50 \mathrm{keV}, 50-100 \mathrm{keV}$, and 100-300 keV energy channels show three HXR sources: S1, $\mathrm{S} 2$, and S3 (Figs. 4b, 5c, and d). S1 and S2 are located at the outer edges of $\mathrm{H} \alpha$ ribbons $\mathrm{B} 1$ and $\mathrm{B} 2$ on the two sides of the magnetic PIL in the early phase (Fig. 5) and spatially consistent with the two ends of the TRACE $195 \AA$ loop. In the late phase, the distance between S1 and S2 has increased, and S2 is still at the outer edge of $\mathrm{B} 2$, while $\mathrm{S} 1$ is in the B1 ribbon. We propose the following scenario. Hotter loops are formed under the reconnection site. They are cooling and forming cooler loops visible 

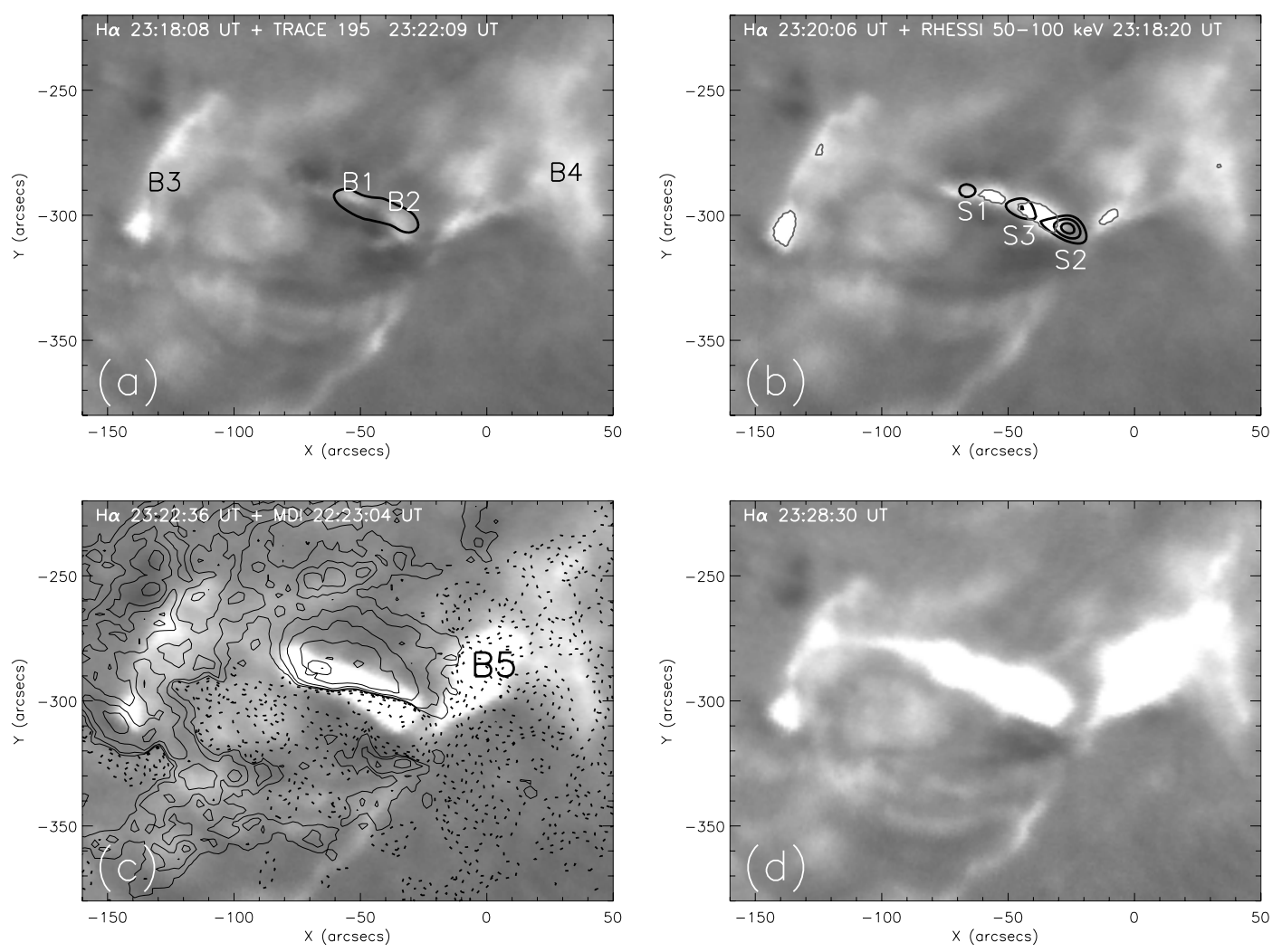

Fig. 4. $\mathrm{H} \alpha$ line-center filter images of the flare observed at HSOS at different times as shown in each image showing the quick extension of the ribbon B1 and B2, which even merge. B5 is very bright after 22:23 UT. The superposed contours in a) show the TRACE $195 \AA$ image at 23:22:09 UT. Superposed on b) are the RHESSI image at 23:18:20 UT in 50-100 keV (black contour) and the H $\alpha$ image at 23:18:08 UT (gray contour, showing the initial $\mathrm{H} \alpha$ brightenings in a)), respectively. The contour level for TRACE $195 \AA$ image is 55\% of the maximum value of the image, while the contour levels for RHESSI image are 50\%, 70\%, and 90\% of the maximum value of the image. The superposed contours in frame c) are MDI LOS field at 22:23:04 UT (solid/dotted contour for positive/negative field). The contour levels are $\pm 50,200,500,1000,1600$, 2200 Gauss.

in $195 \AA$ and in $\mathrm{H} \alpha$ (ribbons B1 and B2). As the reconnection point is rising, which is visualized by the separating motion of the HXR sources S1 and S2, the cool loops are lower than the hot loops, as suggested by the respective positions of the footpoints of the loops, B1 and B2 compared with S1 and S2 (Schmieder et al. 1996). S1 and S2 are clearly seen only in the periods of 23:18:20 UT-23:19:00 UT and 23:19:40 UT-23:20:20 UT in the harder channel. These facts lead to the conclusion that S1 and S2 are two HXR footpoint sources of the small hot loop. S3 could be a loop-top HXR source located at the reconnection site.

\subsection{HXR spectrum and flare energy}

To study the HXR spectrum and the thermal and nonthermal energy content in the flare, we conducted energy spectrum analysis from RHESSI data. We first retrieved energy spectrum data from RHESSI observation and then analyzed the energy spectrum using a model consisting of a thermal component (Brown et al. 1979) and a nonthermal thick-target component (Brown $1971,1972)$ to fit the observed energy spectrum. The energy spectra were retrieved for 167 energy bins from detectors $1 \mathrm{~F}$, $3-6 \mathrm{~F}$, and $8-9 \mathrm{~F}$ with the pileup correction enabled. Each energy bin represents $1 \mathrm{keV}, 2 \mathrm{keV}$, and $5 \mathrm{keV}$ in the energy ranges 3-100 keV, 100-200 keV, and 200-300 keV, respectively. The time interval used was $20 \mathrm{~s}$. Therefore, we have 120 time bins in the period from 23:14:00 to 23:54:00 UT. The background model was selected for each energy range to allow for variation with time. We chose linear extrapolations between the nighttime count rates (before and after the flare) for the lower energy ranges (3-50 keV) and higher order (order 3) fits for the energy bands above $50 \mathrm{keV}$ (Li et al. 2005). During the fit process, both the broken energy and the high-energy cutoff of the electrons were set to $10 \mathrm{MeV}$ (equivalent to a single power law) while the low-energy cutoff $E_{\text {cutoff }}$ was taken to be a free parameter, i.e., to vary from time to time. From the fit, we obtained a set of parameters including emission measure $E M$, temperature $T$, spectral index $\gamma$, and total electrons $n_{a}$ above the low-energy cutoff $E_{\text {cutoff }}$. The obtained $E_{\text {cutoff }}$ in our fit varies in the range of $17-28 \mathrm{keV}$ with an average of $21 \mathrm{keV}$, which is consistent with the generally accepted value (20 keV) (e.g., Veronig et al. 2005). The time profile of $E_{\text {cutoff }}$ is plotted in Fig. 8 .

The simulated energy spectra, together with the observed ones for the above-mentioned three peaks (P1-P3), are shown in Fig. 9. The indices, inferred from the fitting, of the power-law distribution of nonthermal electrons for the three peaks are 7.7, 5.0, and 5.0, respectively. This indicates that spectra for P2 and $\mathrm{P} 3$ are much harder than $\mathrm{P} 1$, suggesting the hardening of electron spectrum during the increasing phase. It is possibly because more energy was released at the later two peak times, which accelerated the electrons to higher energy. Even though P2 and P3 have a time difference of about $80 \mathrm{~s}$, they have a similar spectral index, presumably implying that the intermittent reconnections at these two times have equivalent outcomes.

Based on the fitting parameters above, we used the formulae given in $\mathrm{Li}$ et al. (2005) to compute the thermal energy $E_{\text {therm }}$, 

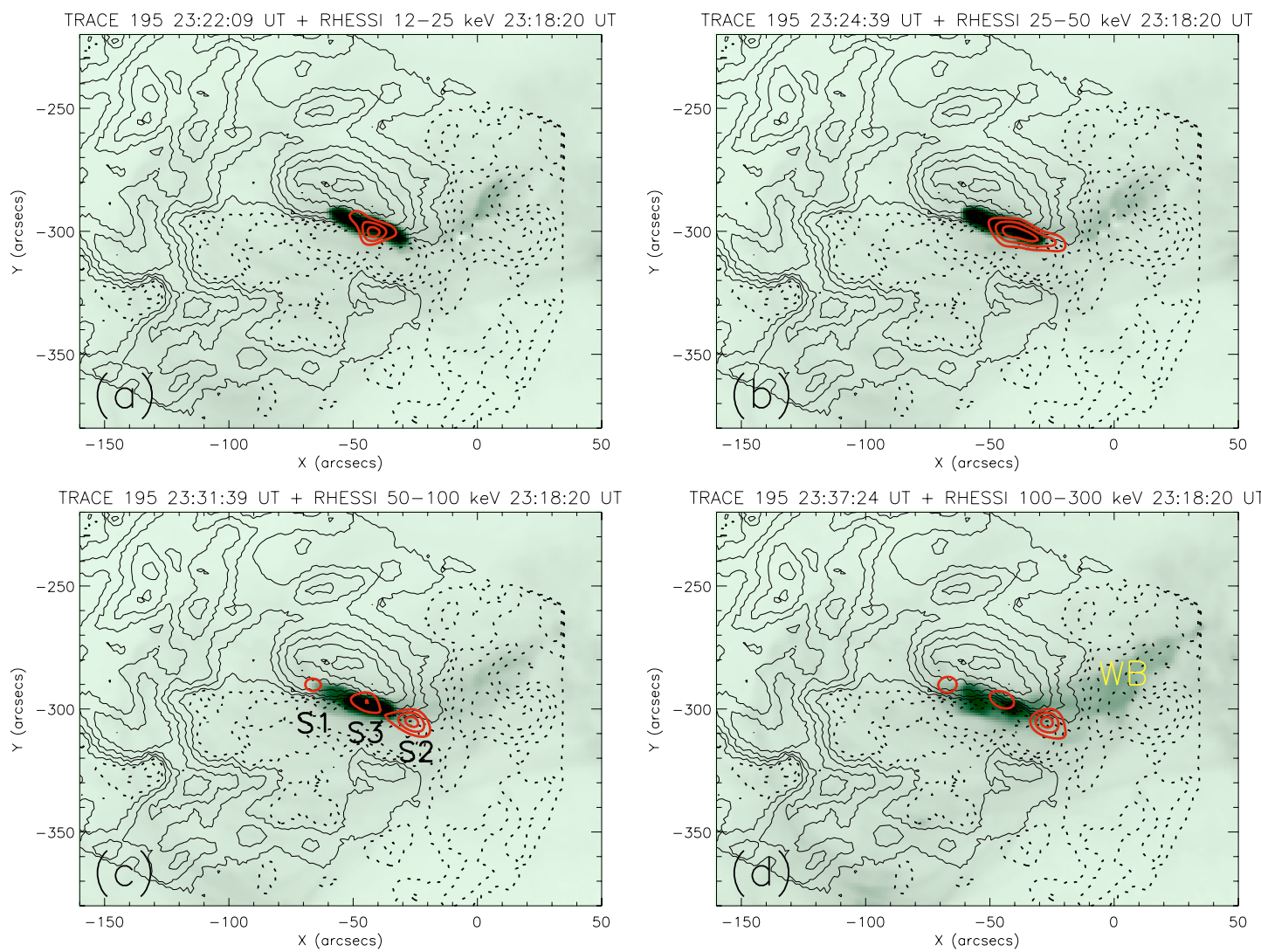

Fig. 5. RHESSI images (red contours) at 23:18:20 UT on September 13 of four energy channels a) 12-25 keV, b) 25-50 keV, c) 50-100 keV, and d) 100-300 keV, respectively, overlaid on TRACE $195 \AA$ images at a) 23:22:09 UT, b) 23:24:39 UT, c) 23:31:39 UT, and d) 23:37:24 UT. Contours of the Huairou LOS magnetic field at 23:12:34 UT of September 13 are superposed. The contour levels of RHESSI image are 50\%, 70\%, and $90 \%$ of the maximum value of the image, and the contour levels of the magnetic field are $\pm 50,200,500,1000,1600,2200 \mathrm{G}$. Solid/dotted contours stand for positive/negative fields. Notice the enhancement of the brightening (marked "WB" in frame d)) on the west side of the tiny loop at 23:37:24 UT in the TRACE images.

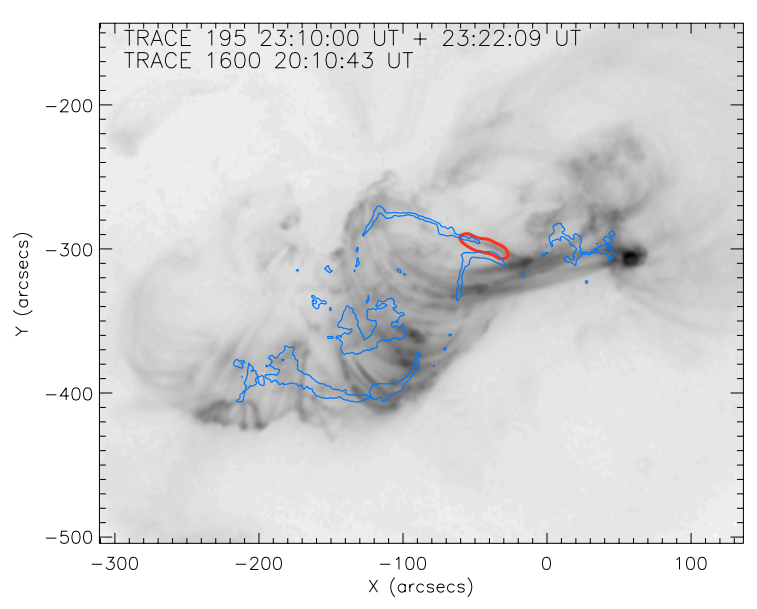

Fig. 6. TRACE $195 \AA$ image of the previous X1.5 flare at 23:10:00 UT is superposed with TRACE $1600 \AA$ image at 20:10:43 UT (thin contours) and TRACE $195 \AA$ image of the studied flare at 23:22:09 UT (thick contour).

nonthermal energy flux $F_{\text {ntherm }}$ of the flare. We used the formula $V=A^{3 / 2}$ (Emslie et al. 2004) to compute the volume $V$ of thermal plasma from the area $A$ of the $50 \%$ contour of RHESSI 12-25 keV images as done by Emslie et al. (2004). The total

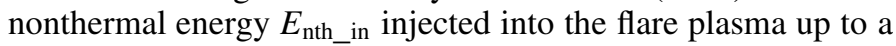
given time was obtained by integrating $F_{\text {ntherm }}$ over time from the flare onset to that time. The computed energy and flux are plotted in Fig. 8. It is shown in the figure that the total injected nonthermal energy is about $2.65 \times 10^{32} \mathrm{erg}$. The total energy (total nonthermal energy plus maximum thermal energy) in the flare has a similar value because the maximum value of $E_{\text {therm }}$ is two orders of magnitude lower than the nonthermal energy. It is worth mentioning that the energy contents are estimated based on the spectral fitting in limited energy ranges (higher than the lowest RHESSI detectable energy, i.e., $3 \mathrm{keV}$ ).

\subsection{Associated coronal mass ejection}

The long-duration triple X-ray event can be divided into two episodes. First, a complex (two peaks) X-ray flare (at about S09E10) between 19:19-20:57 UT with peak emission at 19:27 UT (X1.5) and 20:04 UT (X1.4). And then, the X1.7 X-ray flare studied in this paper occurred at about S10E03 between 23:15-23:30 UT with peak emission at 23:22 UT.

The first episode was followed by a front-sided, strong, and complex asymmetric full halo CME. Nevertheless, only a very faint CME was associated with the second episode (the $\mathrm{X} 1.7$ flare studied in this paper) at the position angle $170^{\circ}$. This faint CME was seen in the FOV of LASCO/C2 coronagraph from 23:36 UT of September 13 to 00:12 UT of September 14 and in the FOV of LASCO/C3 from 00:18 UT to 01:18 UT of September 14. 


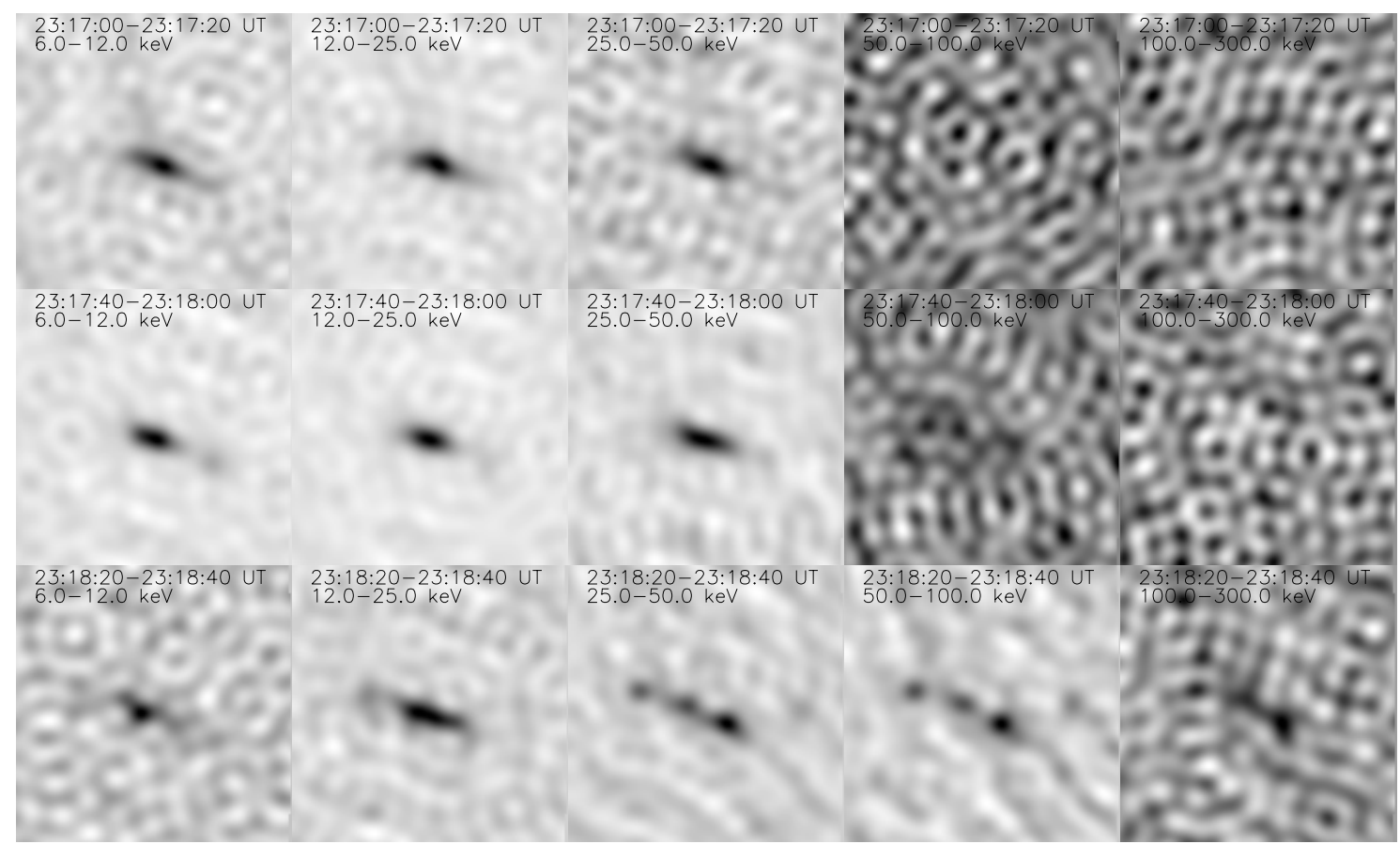

Fig. 7. Reconstructed RHESSI images in the energy channels of 6-12 keV (first column), 12-25 keV (second column), 25-50 keV (third column), 50-100 keV (fourth column), and 100-300 keV (fifth column) in the time ranges of 23:17:00-23:17:20 UT (first row), 23:17:40-23:18:00 UT (second row), and 23:18:20 UT-23:18:40 UT (third row). The images are reconstructed from collimators and detectors $3 \mathrm{~F}-6 \mathrm{~F}$ and $8 \mathrm{~F}$ using the CLEAN image algorithm with $20 \mathrm{~s}$ time interval. Notice the fast extension of the sources between 23:17 UT and 23:18:40 UT in the high-energy channel.

We adopted the CME parameters from the CME catalogue described in Yashiro et al. (2004). The linear fit to the height profile gives a constant velocity of $1000 \mathrm{~km} \mathrm{~s}^{-1}$, while the 2 nd order fit gives a velocity of $865 \mathrm{~km} \mathrm{~s}^{-1}$ at 20 solar radii with an acceleration of $-14 \mathrm{~m} \mathrm{~s}^{-2}$. It is worth mentioning that the velocity and acceleration have large uncertainty because the CME is very weak leading to poor height estimates.

\section{Magnetic field extrapolation}

\subsection{Method}

To understand the origin of the flare and particularly the precise loci of possible energy release in this complex magnetic configuration, we performed extrapolations of the magnetic field observed in the photosphere before and after the flare using two methods, the CFF method assuming a potential field and the NLFFF approach. Before the X1.7 flare, a large post-flare loops system was observed in the high corona (Fig. 3). The loops are well-reproduced with the CFF method using the LOS field observed with MDI (Fig. 10a). This indicates that the high corona is in a potential state at 22:23 UT of 2005 September 13. There are no stressed field lines in the high corona that can produce the X1.7 flare. The energy should come from below. We noticed that the shear and twist above the $\delta$ spot was large and increasing (Fig. 2). Therefore, we conducted extrapolations under an assumption of an NLFFF configuration using the optimized upward integration method developed by Song et al. (2006, 2007) for Huairou data (HVVM) at two times, i.e., 23:13 UT of 2005 September 13 (just before the X1.7 flare) and 01:39 UT of 2005 September 14 (two hours after the flare).

Our optimized upward integration NLFFF computation method is based on the equations $\nabla \cdot \boldsymbol{B}=0$ and $\boldsymbol{J} \times$ $\boldsymbol{B}=0$ (Lorentz force). By using the semi-separability of field

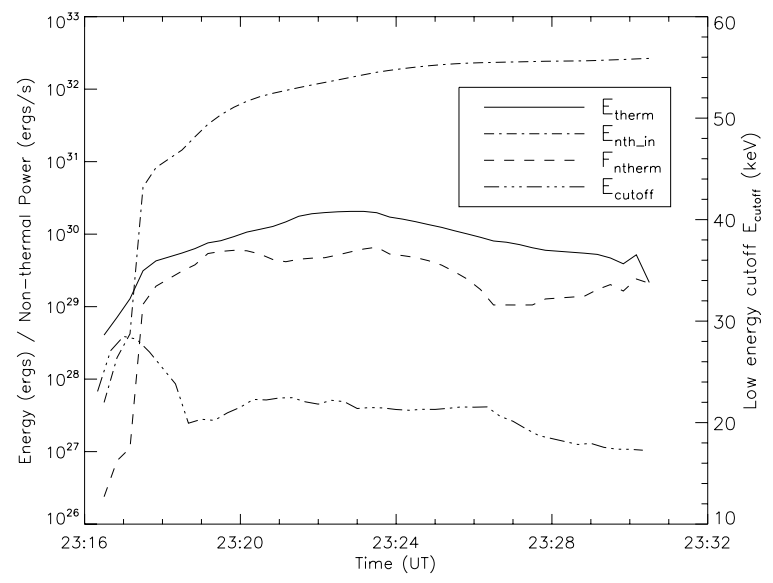

Fig. 8. Time profiles of thermal energy, nonthermal energy flux, total input nonthermal energy, and low-energy cutoff of the power-law distribution of nonthermal electrons derived from RHESSI spectrum analysis. The total energy in the flare is $2.69 \times 10^{32}$ erg if we take the maximum $E_{\text {therm }}$ as the thermal energy content in the flare.

functions, these partial differential equations are reduced to a set of solvable ordinary differential equations. The Runge-Kutta method with double precision gives the field values very fast and accurately, layer by layer, by means of smooth function manipulation. The boundary conditions at the bottom layer used in our computation are the three components of the observed vector magnetic field in the photosphere. The boundary conditions at the four lateral surfaces and top boundary are provided by the potential field model computation using the LOS component of the observed vector magnetic field. This method showed a good correspondence between the computed field lines and the 

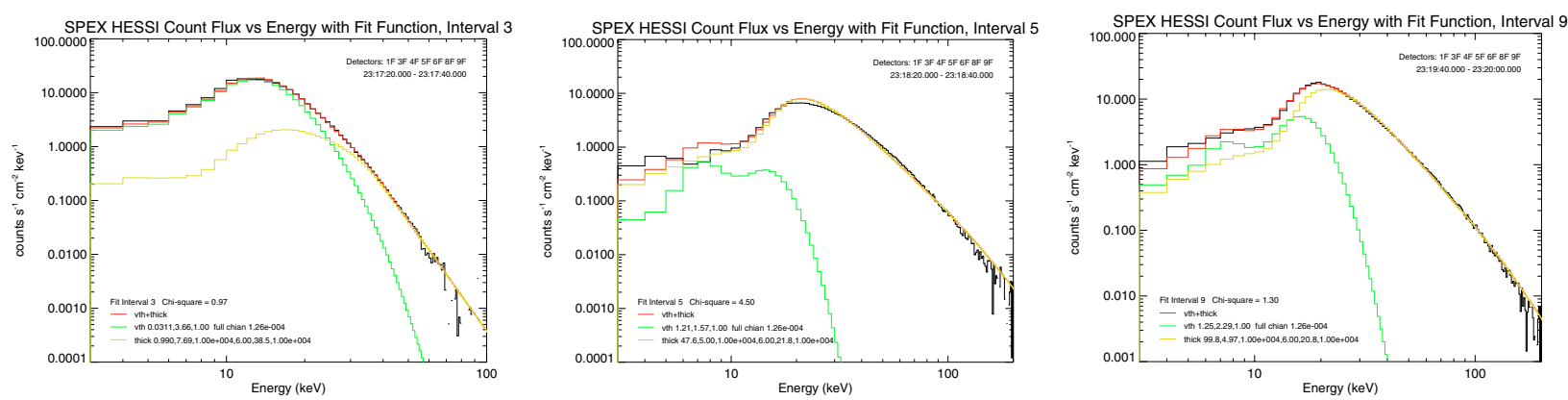

Fig. 9. Observed and fitted RHESSI energy spectrum for the three peaks P1-P3. The fit was done with a model consisting of a thermal component and a nonthermal thick-target component. The energy spectra are retrieved from detectors $1 \mathrm{~F}, 3-6 \mathrm{~F}$, and 8-9F with $20 \mathrm{~s}$ time interval. See Sect. 4.3 for more details.

EIT $195 \AA$ A configurations. Readers are referred to Song et al. $(2006,2007)$ for more details about this method.

\subsection{Results from NLFFF extrapolations}

A small number of selected field lines computed from the data of NLFFF extrapolation are plotted in Fig. 10b and c for data before and after the flare, respectively. The figures show the complicated magnetic configuration above this active region and the possible changes in magnetic connections during the flare process. There are many distorted and shortened low-lying field lines connecting the two main polarities of the $\delta$ spot, as found by Zirin \& Liggett (1987) when they studied the morphology of $\delta$ spots. Such distorted low-lying short loops show the stress of the magnetic field and substantially increase the magnetic free energy. One significant feature of the magnetic connectivity of this region is that, in the region of the positive polarity, only the field lines close to the PIL are connected to the negative polarity of the $\delta$ spot. The flare studied in this paper just took place around the PIL. The field lines in Fig. 10b and c are plotted from the same starting locations in the positive polarities. To do this, we carefully coaligned the magnetograms at the two times using the corresponding photospheric (sunspot) images. The HSOS magnetic field data have a pixel resolution of $0.35^{\prime \prime}$, leading to a coalignment accuracy of $0.7^{\prime \prime}$.

Figure 10 shows that there are several magnetic systems. A magnetic system (M1) existing before the flare (Fig. 10b) connects the main positive polarity in the eastern part of the region to the main negative polarity in the western part. Then in the northern part of the region, we identified a magnetic system (M3) connecting the positive polarity of the $\delta$ spot and the negative polarity in the western part. Figure 10c represents the new loops after the flare. There is a low-lying system (M2), which connects the main positive and negative polarities of the $\delta$ spot. The M1 system has highly sheared loops, while M2 system has more potential loops.

By comparing Fig. 10 with Figs. 4 and 5, the field lines of the systems M1 and M2 connect the two $\mathrm{H} \alpha$ ribbons (B1 and B2) and the two HXR footpoint sources (S1 and S2) (see the 2D sketch in Fig. 10d). Field lines starting from the positive polarity in the northeast part of the region are connected to a region far away (system M3).

By comparing the field lines in Fig. 10b with that in Fig. 10c, we can clearly see the changes in the magnetic connectivity during the flare process, which are explicitly illustrated by the red lines in the figure. We speculate that there was a magnetic reconnection among the above-mentioned magnetic systems. This could happen due to the successive flux emergence and continuous shear motion in the vicinity of the $\delta$ spot (Fig. 3), and these rising low-lying magnetic systems, M1, visualized by the quick expansion of $\mathrm{H} \alpha$ ribbons (B1 and B2) and the separating motion of HXR footpoint sources (S1 and S2). The interaction site could correspond to the HXR loop-top source S3 (Figs. 4, 5, and 7).

\section{Discussion and conclusion}

We studied the X1.7 flare of 2005 September 13 that occurred in a flare-productive active region (NOAA 10808) during a coordinated observation program (JOP178) with space-borne and ground-based instruments, including GOES, RHESSI, SOHO/MDI, TRACE, THEMIS/MTR, HSOS/HVVM. Our results show that the previous X1.5 quadrupolar flare decreased the magnetic nonpotentiality in the high corona, while the nonpotentiality in the low corona above the $\delta$ spot was still increasing. Continuous large-scale magnetic flux tube emergence and shear motion in the active region increase the magnetic shear and twist in the region and lead to the rise of the low-lying magnetic system, which then interacts with the over-lying magnetic systems leading to magnetic reconnections. NLFFF extrapolation clearly shows the change in magnetic connectivity after the reconnection process. Thermal and nonthermal energy computation based on RHESSI energy-spectrum analysis indicates that the total nonthermal energy input into this flare is more than two orders of magnitude higher than the maximum thermal energy, indicating that this flare is of nonthermal origin.

The studied flare occurs in the decreasing phase of a previous long-duration event according to the GOES X-ray flux. RHESSI light curves in low-energy channels are consistent with GOES X-ray flux, they both present two peaks. However, RHESSI light curves in high-energy channels $(>50 \mathrm{keV})$ clearly show two peaks, which precede the peak times in the low-energy channels by 20-30 s. RHESSI images show three HXR emission sources (S1, S2, and S3). Both S1 and S2 are located in opposite magnetic polarities and are spatially consistent with the two ends of TRACE $195 \AA$ loop (Fig. 5) and the two H $\alpha$ ribbons, B1 and B2 (Fig. 4). Therefore, we conclude that S1 and S2 are footpoint sources. The location of S3 suggests that it is a loop-top source. The three HXR sources form a configuration similar to the Masuda flare (Masuda et al. 1994). S1 and S2 are located at the external edge of B1 and B2, suggesting that the X-ray and TRACE loops are formed after reconnection and progressively cooled. They are the cool plasma loop signatures ( $\mathrm{H} \alpha$ ribbons). Such a scenario was confirmed by the magnetic configuration analysis of the region. In the low corona above the $\delta$ spot, the magnetic twist and shear are still strong (Fig. 2). Therefore we 

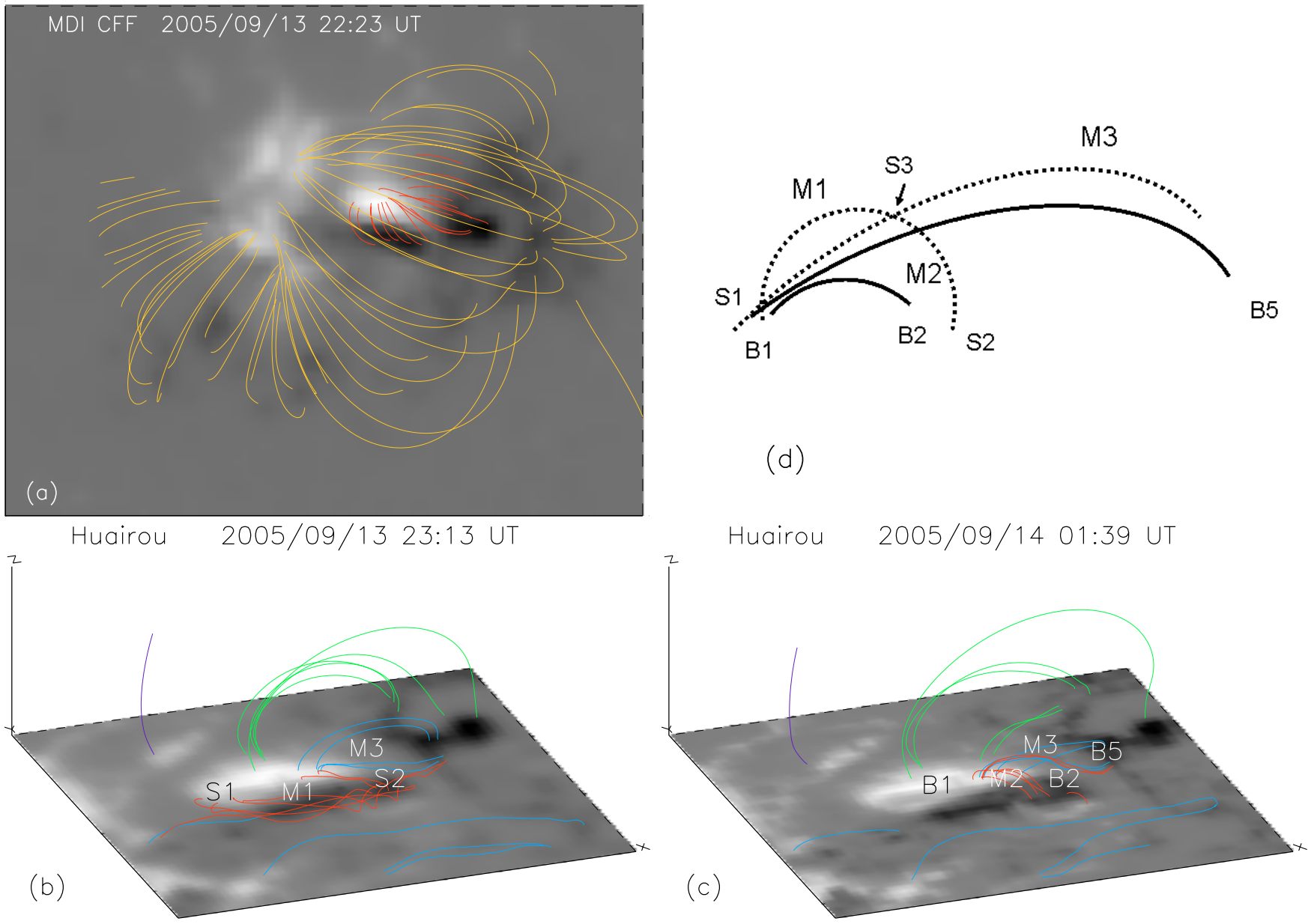

Fig. 10. a) Extrapolated magnetic field lines using the LOS magnetic field (shown as background image) from MDI at 22:23 UT of 2005 September 13 with potential field configuration (observer view); b) and c) Computed field lines from NLFFF extrapolations using vector magnetic field obtained with HVVM at HSOS at 23:13 UT of September 13 and 01:39 UT of 2005 September 14, respectively; d) sketch of the loop systems, $\mathrm{H} \alpha$ bright patches, and HXR sources of the studied flare. The red lines in $\mathbf{b}$ ) and $\mathbf{c}$ ) indicate the field lines, which changed their connections during the flare. Letters "S1"-"S3", "M1"-“M3", and "B1", "B2" and "B5" indicate the locations of HXR footpoint sources, magnetic systems, and H $\alpha$ ribbons, respectively, which are described in the text.

performed the analysis by extrapolating the vector magnetic field of the photosphere from HVVM at HSOS using an NLFFF approach. The NLFFF extrapolations demonstrate the existence of several magnetic systems M1, M2, M3. The system M2 computed after the flare is less sheared than system M1. We propose the sketch shown in Fig. 10d.

The NLFFF extrapolations depend strongly on the boundary conditions, which involves the photospheric transverse magnetic field. It is well known that the determination of the transverse field is difficult due to the problems arising from the $180^{\circ}$ ambiguity of the transverse field and the Faraday effect ( $\mathrm{Su}$ et al. 2006). In our case, we applied the algorithm described in Wang et al. (2001) to our data to remove the $180^{\circ}$ ambiguity. This method cannot completely resolve the problem as reviewed by Metcalf et al. (2006). We can still see some inconsistencies at a few points in Fig. 10b; nevertheless, we believe that this does not change our results as a whole.

The HXR emission is recorded up to $200 \mathrm{keV}$ for this flare in the period of 23:18 UT-23:20 UT and to less than $100 \mathrm{keV}$ for other times. We analyzed the HXR energy spectrum using a model consisting of a thermal component and a thick-target nonthermal component. Based on the fit parameters, we estimate the thermal energy and nonthermal flux of the flare at different times and the total nonthermal energy injected into the flare plasma. If we adopt the mean lifetime $t_{a}=1.0 \mathrm{~s}$ for the high-energy electrons (Emslie 1983; Li et al. 2005) and bear in mind the uncertainty in the energy estimation discussed below, the nonthermal energy $E_{\text {ntherm }}\left(=F_{\text {ntherm }} \times t_{a}\right)$ and the thermal energy $\left(E_{\text {therm }}\right)$ are comparable (Fig. 8). On the other hand, the total nonthermal energy injected into the flare plasma $\left(E_{\text {nth_in }}\right)$ is more than two orders of magnitude higher than the maximum thermal energy (see Fig. 8). In other words, the flare has a nonthermal property. Similar cases are reported by other authors (Saint-Hilaire \& Benz 2002).

It is commonly accepted that the low-energy cutoff ( $\left.E_{\text {cutoff }}\right)$ of nonthermal electron distribution is critical in the estimation of nonthermal energy in a flare, due to its power-law property. Increasing/decreasing $E_{\text {cutoff }}$ by a few $\mathrm{keV}$ will remove/add a large amount of nonthermal energy to the estimated value (McDonald et al. 1999). However, it is quite difficult to determine the low-energy cutoff $E_{\text {cutoff }}$ from observations, so in our fitting process, $E_{\text {cutoff }}$ is allowed to change with time, which yields an average value of $21 \mathrm{keV}$. The inaccuracy of $E_{\text {cutoff }}$ could introduce uncertainties into our analysis. The measurement accuracy of the HXR source volume $V$ will affect the estimation of thermal energy. In this case, the volume may differ by a factor of 2 , resulting in about a $40 \%$ uncertainty for the estimated thermal energy (Li et al. 2005). 
Acknowledgements. The authors are grateful to the RHESSI Team for free access to the RHESSI data and the development of the software and to the THEMIS team, which operates the telescope at Tenerife. We thank P. Mein for his help in THEMIS data processing. CME data used in this paper is generated and maintained at the CDAW Data Center by NASA and The Catholic University of America in cooperation with the Naval Research Laboratory. SOHO is a project of international cooperation between ESA and NASA. THEMIS is a French-Italian telescope operated on the island of Tenerife by the CNRS-CNR in the Spanish Observatorio del Teide of the Instituto de Astrofísica de Canarias. The work of HL was supported by the National Natural Science Foundation of China (NSFC, grant number 10573038 and 10333040), National Basic Research Program of China (2006CB806302), and CAS Project KJCX2-YW-T04.

\section{References}

Ai, G. X., \& Hu, Y. F. 1986, Publ. Beijing Astron. Obs., 8, 1

Ai, G.-X., Li, W., \& Zhang, H.-Q. 1982, Chin. Astron. Astrophys., 6, 129

Alissandrakis, C. E. 1981, A\&A, 100, 197

Aly, J. J. 1992, Sol. Phys., 138, 133

Aulanier, G., DeLuca, E. E., Antiochos, S. K., McMullen, R. A., \& Golub, L. 2000, ApJ, 540, 1126

Aulanier, G., Pariat, E., \& Démoulin, P. 2005, A\&A, 444, 961

Aulanier, G., Pariat, E., Démoulin, P., \& Devore, C. R. 2006, Sol. Phys., 238, 347

Berger, M. A. 1984, Geophys. Astrophys. Fluid Dyn., 30, 79

Berlicki, A., Schmieder, B., Vilmer, N., Aulanier, G., \& Del Zanna, G. 2004, A\&A, 423, 1119

Bommier, V., Landi Degl'Innocenti, E., Landolfi, M., \& Molodij, G. 2007, A\&A, 464, 323

Brown, J. C. 1971, Sol. Phys., 18, 489

Brown, J. C. 1972, Sol. Phys., 26, 441

Brown, J. C. \& MacKinnon, A. L. 1985, ApJ, 292, L31

Brown, J. C., Spicer, D. S., \& Melrose, D. B. 1979, ApJ, 228, 592

Chen, P. F., \& Shibata, K. 2000, ApJ, 545, 524

Chiu, Y. T., \& Hilton, H. H. 1977, ApJ, 212, 873

Datlowe, D. W., \& Lin, R. P. 1973, Sol. Phys., 32, 459

Démoulin, P., Bagalá, L. G., Mandrini, C. H., Hénoux, J. C., \& Rovira, M. G. 1997, A\&A, 325, 305

Deng, Y., Ai, G., Wang, J., et al. 1997, Sol. Phys., 173, 207

Domingo, V., Fleck, B., \& Poland, A. I. 1995, Sol. Phys., 162, 1

Emslie, A. G. 1983, ApJ, 271, 367

Emslie, A. G., Kucharek, H., Dennis, B. R., Gopalswamy, N., \& Holman, G. D. 2004, J. Geophys. Res., 109, A10104

Falconer, D. A. 2001, J. Geophys. Res., 106, 25185

Fletcher, L., López Fuentes, M. C., Mandrini, C. H., et al. 2001a, Sol. Phys., 203, 255

Fletcher, L., Metcalf, T. R., Alexander, D., Brown, D. S., \& Ryder, L. A. 2001b, ApJ, 554, 451

Galsgaard, K., \& Parnell, C. E. 2005, A\&A, 439, 335

Galsgaard, K., Parnell, C. E., \& Blaizot, J. 2000, A\&A, 362, 395

Hagyard, M. J., Teuber, D., West, E. A., \& Smith, J. B. 1984, Sol. Phys., 91, 115

Handy, B. N., Acton, L. W., Kankelborg, C. C., et al. 1999, Sol. Phys., 187, 229

Landolfi, M., Arena, P., \& Deglinnocenti, E. L. 1984, Sol. Phys., 93, 269

Leka, K. D., \& Barnes, G. 2003a, ApJ, 595, 1277

Leka, K. D., \& Barnes, G. 2003b, ApJ, 595, 1296
Li, H. 2002, Chin. J. Astron. Astrophys., 2, 174

Li, H., \& Li, Y. P. 2007, Adv. Space Res., 40, in press

Li, H., Sakurai, T., Ichimoto, K., \& UeNo, S. 2000a, PASJ, 52, 483

Li, H., Sakurai, T., Ichimoto, K., \& UeNo, S. 2000b, PASJ, 52, 465

Li, H., Berlicki, A., \& Schmieder, B. 2005, A\&A, 438, 325

Lin, R. P., Dennis, B. R., Hurford, G. J., et al. 2002, Sol. Phys., 210, 3

López Ariste, A., Rayrole, J., \& Semel, M. 2000, A\&AS, 142, 137

López Ariste, A., Aulanier, G., Schmieder, B., \& Sainz Dalda, A. 2006, A\&A, 456,725

López Fuentes, M. C., Demoulin, P., Mandrini, C. H., \& van Driel-Gesztelyi, L. 2000, ApJ, 544, 540

MacKinnon, A. L., \& Brown, J. C. 1984, A\&A, 132, 229

Masuda, S., Kosugi, T., Hara, H., Tsuneta, S., \& Ogawara, Y. 1994, Nature, 371, 495

McDonald, L., Harra-Murnion, L. K., \& Culhane, J. L. 1999, Sol. Phys., 185, 323

Metcalf, T. R., Jiao, L., McClymont, A. N., Canfield, R. C., \& Uitenbroek, H. 1995, ApJ, 439, 474

Metcalf, T. R., Leka, K. D., Barnes, G., et al. 2006, Sol. Phys., 237, 267

Miyagoshi, T., \& Yokoyama, T. 2004, ApJ, 614, 1042

Moon, Y.-J., Choe, G. S., Yun, H. S., Park, Y. D., \& Mickey, D. L. 2002, ApJ, 568,422

Nagashima, K., Isobe, H., Yokoyama, T., et al. 2007, ApJ, submitted

Nandy, D., Hahn, M., Canfield, R. C., \& Longcope, D. W. 2003, ApJ, 597, L73

Parker, E. N. 1989, Sol. Phys., 121, 271

Parnell, C. E., \& Galsgaard, K. 2004, A\&A, 428, 595

Rayrole, J., \& Mein, P. 1993, in The Magnetic and Velocity Fields of Solar Active Regions, ed. H. Zirin, G. Ai, \& H. Wang, IAU Colloq. 141, ASP Conf. Ser., 46,170

Régnier, S., \& Canfield, R. C. 2006, A\&A, 451, 319

Régnier, S., \& Priest, E. R. 2007, A\&A, 468, 701

Saint-Hilaire, P., \& Benz, A. O. 2002, Sol. Phys., 210, 287

Sakurai, T. 1982, Sol. Phys., 76, 301

Scherrer, P. H., Bogart, R. S., Bush, R. I., et al. 1995, Sol. Phys., 162, 129

Schmidt, H. U. 1964, in The Physics of Solar Flares, ed. W. N. Hess, 107

Schmieder, B., \& van Driel-Gesztelyi, L. 2005, in IAU Symp. 226, ed. K. Dere,

J. Wang, \& Y. Yan, 149

Schmieder, B., Heinzel, P., van Driel-Gesztelyi, L., \& Lemen, J. R. 1996, Sol. Phys., 165, 303

Semel, M., \& Rayrole, J. 1968, in Structure and Development of Solar Active Regions, ed. K. O. Kiepenheuer, IAU Symp., 35, 134

Song, M. T., Fang, C., Tang, Y. H., Wu, S. T., \& Zhang, Y. A. 2006, ApJ, 649, 1084

Song, M. T., Fang, C., Zhang, H. Q., et al. 2007, ApJ, in press

Su, J. T., Zhang, H. Q., Deng, Y. Y., et al. 2006, ApJ, 649, L141

Taylor, J. B. 1986, Rev. Mod. Phys., 58, 741

Titov, V. S., Galsgaard, K., \& Neukirch, T. 2003, ApJ, 582, 1172

Veronig, A. M., Brown, J. C., Dennis, B. R., et al. 2005, ApJ, 621, 482

Wang, H., Yan, Y., \& Sakurai, T. 2001, Sol. Phys., 201, 323

Wang, H. M., Liu, C., Jing, J., \& Yurchyshyn, V. 2007, ApJ, submitted

Wang, J., Shi, Z., Wang, H., \& Lue, Y. 1996, ApJ, 456, 861

Yashiro, S., Gopalswamy, N., Michalek, G., et al. 2004, J. Geophys. Res. (Space Phys.), 109, 7105

Yokoyama, T., \& Shibata, K. 2001, ApJ, 549, 1160

Zirin, H., \& Liggett, M. A. 1987, Sol. Phys., 113, 267 